\title{
Enhancing the axial resolution of quantum optical coherence tomography by chirped quasi-phase matching
}

\author{
Silvia Carrasco, Juan P. Torres, and Lluis Torner
}

ICFO-Institut de Ciencies Fotoniques and Department of Signal Theory and Communications, Universitat Politecnica de Catalunya, 08034 Barcelona, Spain

\author{
Alexander Sergienko, Bahaa E. A. Saleh, and Malvin C. Teich \\ Quantum Imaging Laboratory, Department of Electrical and Computer Engineering and Department of Physics, Boston University,
} Boston, Massachusetts 02215

Received April 21, 2004

\begin{abstract}
We show theoretically that a chirped quasi-phase-matching nonlinear crystal structure can significantly enhance the axial resolution in quantum optical coherence tomography by increasing the spectral width of the generated entangled photon pairs. For pulsed pumps we show how the pump-pulse duration affects the maximum resolution attainable. (C) 2004 Optical Society of America

OCIS codes: $110.4500,170.4500,270.0270$.
\end{abstract}

Optical coherence tomography (OCT) has become a versatile, noninvasive imaging technique ${ }^{1,2}$ with many applications in biology and medicine. ${ }^{3-5}$ It is based on an interferometric scheme that uses light of short temporal coherence to section a reflective sample. Because of its interferometric nature, OCT can achieve high axial resolution, independent of focusing conditions, since axial and transverse resolutions are determined independently. ${ }^{6}$ The axial resolution is governed mainly by the temporal coherence length of the light source and thus enhanced by the use of broadband sources, such as superluminescent light-emitting diodes and ultrashort pulsed lasers. ${ }^{7,8}$

In general, however, the presence of chromatic dispersion in the sample degrades the resolution of OCT, since dispersion tends to increase the signal width. ${ }^{9}$ This deleterious effect is exaggerated when ultrahigh resolution is required, because of the use of signals with large spectral width. The effect of dispersion can be reduced by use of a light source with a central optimal wavelength, at which the group-velocity dispersion is negligible, ${ }^{10}$ or appropriate numerical dispersion-compensation schemes. ${ }^{11}$

A quantum version of OCT, called quantum optical coherence tomography (QOCT), has been set forth. ${ }^{12}$ QOCT makes use of pairs of entangled photons generated through the nonlinear optical process of spontaneous parametric downconversion. The frequency anticorrelation inherent in the two-photon state when using a cw pump leads to even-order dispersion cancellation, ${ }^{13}$ and the spectral content, or spectral width, of the downconverted photons determines the axial resolution of QOCT. In this Letter we posit that quasi-phase matching (QPM) can be used to substantially enhance the spectral width of the entangled two-photon state produced in the downconversion process while simultaneously maintaining the frequency anticorrelation.

QPM provides a feasible alternative to conventional phase matching for many optical parametric process applications, and QPM engineering opens up new possibilities. In the quantum domain, tunable frequency entanglement with a largely reduced spectral width can be achieved in counterpropagating geometries, ${ }^{14,15}$ and longitudinally and transversely varying QPM strongly affects the spatiotemporal properties of entangled photon pairs. ${ }^{16,17}$ The merit of a longitudinally chirped QPM is that it permits many different signal and idler photon wavelengths to be phase matched at different positions inside the nonlinear crystal, thus broadening the spectral content of the two-photon state. QPM downconversion with a cw pump offers dispersion-canceled QOCT, which is therefore superior to conventional spectral width enhancement techniques that make use of ultrashort pulses.

We consider a pump beam of frequency $\omega_{p}$ with a Gaussian spectral distribution $E_{p}\left(\omega_{p}\right) \propto \exp \left[-\left(\omega_{p}-\right.\right.$ $\left.\left.\omega_{p}^{0}\right)^{2} T_{0}^{2} / 2\right]$ that illuminates a quadratic nonlinear crystal of length $L$. Here, $\omega_{p}^{0}$ is the central frequency of the pump pulse and $T_{0}$ is the pulse duration. A pair of frequency-entangled photons with equal polarization are generated in a nearly collinear geometry. In QOCT the two photons are detected in coincidence in well-defined spatial modes, which, for simplicity, we assume to be plane waves. For typical interaction times one obtains $\omega_{p}=\omega_{s}+\omega_{i}$, where $\omega_{s, i}$ are the frequencies of the signal and idler photons, respectively. At the output of the nonlinear crystal the two-photon quantum state is ${ }^{18}$

$$
|\Psi\rangle=\int \mathrm{d} \omega_{s} \mathrm{~d} \omega_{i} \Phi\left(\omega_{s}, \omega_{i}\right) \hat{a}_{s}^{\dagger}\left(\omega_{s}\right) \hat{a}_{i}^{\dagger}\left(\omega_{i}\right)|0,0\rangle,
$$

where $|0,0\rangle$ is the vacuum state; $\hat{a}_{s}^{\dagger}$ and $\hat{a}_{i}^{\dagger}$ are the photon creation operators for signal and idler waves, respectively; and the state function is written as $\Phi\left(\omega_{s}, \omega_{i}\right) \propto E_{p}\left(\omega_{s}+\omega_{i}\right) W(\Delta k)$, such that $\int \mathrm{d} \omega_{s} \mathrm{~d} \omega_{i}|\Phi|^{2}=1$. The function $W$ comes from the longitudinally varying phase-matching condition in the $z$ direction. We assume that phase 
matching is achieved along the crystal in such a way that the phase-matching conditions vary longitudinally by use of QPM with a chirped period along the $z$ direction, with local period $p(z)$. Note that in general a similar effect can be obtained, for example, by use of temperature gradients. Thus $W$ is given by $W(\Delta k)=\int_{0}^{L} \mathrm{~d} z \exp (i \Delta k z)$, with $\Delta k=k_{p}\left(\omega_{p}\right)-k_{s}\left(\omega_{s}\right)-k_{i}\left(\omega_{i}\right)-2 \pi / p(z)$. The longitudinal wave numbers for the pump, signal, and idler photons are $k_{p}=\omega_{p} n_{p}\left(\omega_{p}\right) / c, k_{s}=\omega_{s} n_{s}\left(\omega_{s}\right) / c$, and $k_{i}=\omega_{i} n_{i}\left(\omega_{i}\right) / c$.

The entangled two-photon state enters a Hong$\mathrm{Ou}$-Mandel interferometer, modified by placing the sample to be probed in one of the arms and an adjustable temporal delay $\tau$ in the other. The coincidence rate $C(\tau)$, the rate of detection of both the signal and idler photons in coincidence as a function of temporal delay $\tau$, is given by

$$
\begin{aligned}
C(\tau) \simeq & \iint \mathrm{d} \omega_{s} \mathrm{~d} \omega_{i} \mid H\left(\omega_{i}\right) \Phi\left(\omega_{i}, \omega_{s}\right) \\
& -\left.H\left(\omega_{s}\right) \Phi\left(\omega_{s}, \omega_{i}\right) \exp \left[-i\left(\omega_{s}-\omega_{i}\right) \tau\right]\right|^{2},
\end{aligned}
$$

where function $H$ is the transfer function of the sample under study. For convenience, the signal and idler frequencies are written as $\omega_{s}=\omega_{s}^{0}+\Omega_{s}$ and $\omega_{i}=$ $\omega_{i}^{0}+\Omega_{i}$, respectively, where $\omega_{s, i}^{0}$ are the central frequencies and $\Omega_{s, i}$ are the angular frequency deviations about the central frequencies. For the limiting case of long pump-pulse durations (cw limit) relation (2) reduces to the expression given in Abouraddy et al. ${ }^{19}$

We assume that local period $p$ of the grating varies linearly with the propagation distance, i.e., $p(z)=p(0)+(\Delta p / L) z$, so that the total change of the local period between $z=0$ and $z=L$ amounts to $\Delta p$. A sketch of such a crystal is shown in the inset of Fig. 1(a). To reveal the effects on the spectral width of the state function of the chirp alone, let us consider a cw pump, i.e., $E_{p}\left(\omega_{p}\right) \sim \delta\left(\omega_{p}-\omega_{p}^{0}\right)$. The state function $\Phi$ depends only on $\Omega_{s}$, since $\Omega_{s}=-\Omega_{i}$. For the sake of concreteness we consider a quadratic nonlinear crystal: periodically poled potassium titanyl phosphate (PPKTP). The same behavior has been obtained for other crystals, such as lithium niobate. The cw pump wavelength was fixed at $\lambda_{p}^{0}=406 \mathrm{~nm}$ in all cases, and thus $\lambda_{s}^{0}=\lambda_{i}^{0}=812 \mathrm{~nm}$. For such wavelengths, the nominal period $p_{0}$ of the PPKTP grating that phase matches the central wavelengths is $p_{0}=3.51 \mu \mathrm{m}$. To illustrate the behavior of the axial resolution under different QPM configurations, we theoretically consider a sample to be probed [see inset of Fig. 1(b)] formed by two reflection layers, with reflection coefficients $r_{1}$ and $r_{2}$, respectively, separated by a small distance $L_{s}$ and filled with water. ${ }^{20}$ The transfer function of such a sample is given by $H(\omega)=r_{1}+r_{2} \exp \left[2 i n(\omega) L_{s} / c\right]$. The sample considered here is representative of many living structures, composed of a liquid medium, mainly water, and contained inside a membrane. The results obtained here hold for more general structures.

Figures 1 and 2 summarize our main findings. Writing a chirp in the QPM grating leads to broaden- ing of the spectrum of the signal and idler photons. Spectral width $\sigma_{\lambda}$ is shown in Fig. 1(a) as a function of chirp parameter $\Delta p / p_{0}$ for different configurations. In all cases the crystal length was taken to be $L=1 \mathrm{~mm}$. The two curves plotted in Fig. 1(a) correspond to two different locations inside the crystal of the local period that produce phase matching at the central wavelengths, at $z=0$ (open circles), where $p(0)=p_{0}$, and at $z=L / 2$ (filled circles), with $p(L / 2)=p_{0}$. This spectral broadening is directly translated into an improvement in resolution of the QOCT technique. In Fig. 1(b) we plot the resolution, defined as the FWHM of the dip that corresponds to one of the reflective layers in the sample under study. In the figure it is clearly shown how submicrometer resolution can be achieved.

The details of the spectrum and the normalized coincidence interferogram are shown in Fig. 2 for different chirps, corresponding to points $\mathrm{A}, \mathrm{B}, \mathrm{C}$, and $\mathrm{D}$ shown in Fig. 1. In the case that the nominal period is located at $z=0$, i.e., $p(0)=p_{0}$, and the local period increases along the $z$ direction (point $\mathrm{B}$ ), $p(L)>p_{0}$, large spectral widths with peaks outside the central
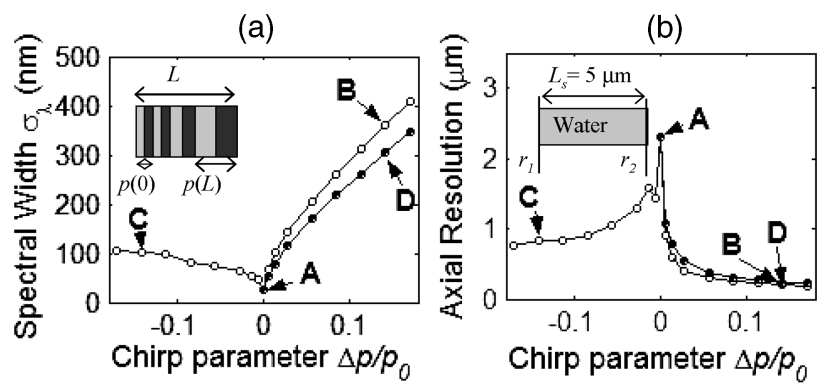

Fig. 1. (a) Calculated spectral width of $|\Phi|^{2}$ and (b) QOCT axial resolution versus chirp parameter $\Delta p / p_{0}$ for cw pump excitation at $\lambda_{p}=412 \mathrm{~nm}$. The twin-photon source is taken to be a PPKTP crystal of length $L=1 \mathrm{~mm}$ with chirp parameter $\Delta p / p_{0}$. The open circles indicate calculations for $p(0)=p_{0}, p(L)=p_{0}+\Delta p$. The filled circles are calculations for $p(0)=p_{0}-\Delta p / 2, p(L)=p_{0}+\Delta p / 2$. The hypothetical sample under study is formed by two membranes with reflection coefficients $r_{1}=r_{2}=0.2$ separated by a distance $L_{s}=5 \mu \mathrm{m}$ and filled with water. The insets in (a) and (b) are sketches of the chirped QPM crystal and the probed sample, respectively.

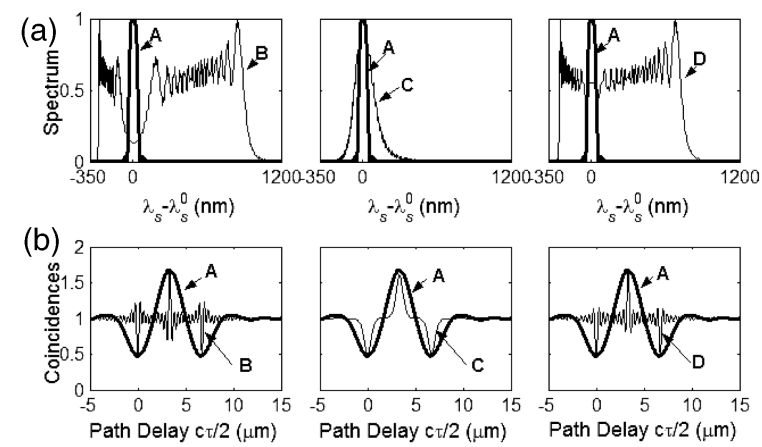

Fig. 2. (a) Calculated spectrum $|\Phi|^{2}$ and (b) coincidence interferogram for the points labeled in Fig. 1. Results for a uniform crystal (A) are shown in all the figures for easy comparison as a thick curve; $\mathrm{B}, p(0)=p_{0}, \Delta p=0.5 \mu \mathrm{m}$; $\mathrm{C}, p(0)=p_{0}, \Delta p=-0.5 \mu \mathrm{m} ; \mathrm{D}, p(L / 2)=p_{0}, \Delta p=0.5 \mu \mathrm{m}$. 


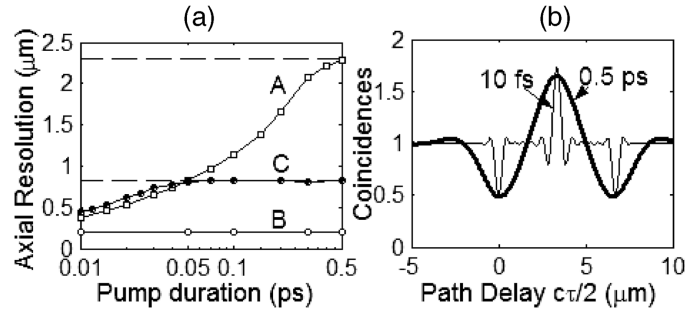

Fig. 3. (a) Axial resolution versus the pump-pulse duration for A, unchirped crystal (squares) and two selected chirped crystals with $\mathrm{B}, p(0)=p_{0}, \Delta p=0.5 \mu \mathrm{m}$ (open circles) and $\mathrm{C}, p(0)=p_{0}, \Delta p=-0.5 \mu \mathrm{m}$ (filled circles). The dashed curves represent the corresponding resolution for $\mathrm{cw}$ excitation in each case. (b) Coincidence interferogram for pulse durations of $0.5 \mathrm{ps}$ and $10 \mathrm{fs}$ in the unchirped crystal case.

frequency are obtained [Fig. 2(a), left column]. This is because, for larger periods of the QPM gratings, wavelengths different from the degenerate ones $\left(\lambda_{s}^{0}=\right.$ $\lambda_{i}^{0}$ ) are also phase matched. The spectrum exhibits oscillatory behavior leading to sidelobes in the coincidence interferogram [Fig. 2(b), left column]. These sidelobes can lead to spurious images of the sample under study. ${ }^{21}$ This problem can be alleviated by use of spectral shapers. ${ }^{6}$ In the case that the local period decreases along the $z$ direction (point $\mathrm{C}$ ), $p(L)<p(0)$, the increase of the spectral width is not as large as in the previous case [Fig. 2(a), central column]. However, the shape of the spectrum is smoother, and thus sidelobes are absent in the coincidence interferogram [Fig. 2(b), central column]. Finally, the right column of Fig. 2 shows the case for which the nominal period is located at the center of the crystal $\left[p(L / 2)=p_{0}\right]$, and the local period along $z$ goes from shorter to larger periods or vice versa (point $\mathrm{D}$ ).

Since the use of short pump pulses also increases the spectral width of the state function of the downconverted photons, we consider the combined effects on axial resolution. However, one must take into account that for pump pulses the even-order dispersion cancellation effect does not hold. In Fig. 3(a) we plot the resolution of a QOCT image as a function of pump-pulse duration $T_{0}$ for an unchirped QPM crystal and for two different chirped crystals. The main conclusion is that the larger the spectral width induced by the chirp, the less important the effect of the pump-pulse duration on axial resolution. One can obtain the same axial resolution with a pump pulse of several femtoseconds in a standard QPM crystal as with a cw pump beam in a properly designed chirped nonlinear crystal. Figure 3(b) compares the coincidence interferogram for two different values of the pump-pulse width. The enhanced resolution offered by the chirped QPM structure is clearly apparent.

In conclusion, we have shown that submicrometer axial resolution of QOCT images can be achieved by use of properly designed chirped QPM crystals as a source of entangled twin-photon pairs. Indeed, the resolution achievable is comparable with the resolution that can be obtained with pump-pulse durations of several femtoseconds in uniform structures, while maintaining the even-order dispersion cancellation effect, which is absent in the femtosecond regime. Because femtosecond sources are one of the key elements for achieving ultrahigh resolution in OCT, the use of appropriately designed chirped QPM nonlinear crystals allows the use of longer pump pulses while maintaining the axial resolution, thereby imposing weaker restrictions on the design of the source.

This work was supported by the Generalitat de Catalunya; by grant BFM2002-2861 from the Government of Spain; by the Center for Subsurface Sensing and Imaging, a U.S. National Science Foundation Engineering Research Center; and by the David and Lucile Packard Foundation. S. Carrasco's e-mail address is carrasco@bu.edu.

\section{References}

1. R. C. Youngquist, S. Carr, and D. E. N. Davies, Opt. Lett. 12, 158 (1987).

2. K. Takada, I. Yokohama, K. Chida, and J. Noda, Appl. Opt. 26, 1603 (1987).

3. A. F. Fercher and E. Roth, Proc. SPIE 658, 48 (1986).

4. D. Huang, E. A. Swanson, C. P. Lin, J. S. Schuman, W. G. Stinson, W. Chang, M. R. Hee, T. Flotte, K. Gregory, C. A. Puliafito, and J. G. Fujimoto, Science 254, 1178 (1991).

5. B. E. Bouma and G. J. Tearney, eds., Handbook of Optical Coherence Tomography (Marcel Dekker, New York, 2002).

6. W. Drexler, J. Biomed. Opt. 9, 47 (2004).

7. W. Drexler, U. Morgner, F. X. Kärtner, C. Pitris, S. A. Boppart, X. D. Li, E. P. Ippen, and J. G. Fujimoto, Opt. Lett. 24, 1221 (1999).

8. A. M. Kowalevicz, T. Ko, I. Harlt, J. G. Fujimoto, M. Pollnau, and R. P. Salathé, Opt. Express 10, 349 (2002), http://www.opticsexpress.org.

9. C. K. Hitzenberger, A. Baumgartner, W. Drexler, and A. F. Fercher, J. Biomed. Opt. 4, 144 (1999).

10. Y. Wang, J. S. Nelson, Z. Chen, B. J. Reiser, R. S. Chuck, and R. S. Windeler, Opt. Express 11, 1411 (2003), http://www.opticsexpress.org.

11. A. F. Fercher, C. K. Hitzenberger, M. Sticker, R. Zawadzki, B. Karamata, and T. Lasser, Opt. Commun. 204, 67 (2002).

12. M. B. Nasr, B. E. A. Saleh, A. V. Sergienko, and M. C. Teich, Phys. Rev. Lett. 91, 0836011 (2003).

13. A. M. Steinberg, P. G. Kwiat, and R. Y. Chiao, Phys. Rev. Lett. 68, 2421 (1992).

14. M. C. Booth, M. Atatüre, G. Di Giuseppe, B. E. A. Saleh, A. V. Sergienko, and M. C. Teich, Phys. Rev. A 66, 023815 (2002).

15. A. De Rossi and V. Berger, Phys. Rev. Lett. 88, 043901 (2002).

16. G. Di Giuseppe, M. Atatüre, M. D. Shaw, A. V. Sergienko, B. E. A. Saleh, and M. C. Teich, Phys. Rev. A 66, 013801 (2002).

17. J. P. Torres, A. Alexandrescu, S. Carrasco, and L. Torner, Opt. Lett. 29, 376 (2004).

18. B. E. A. Saleh, A. F. Abouraddy, A. V. Sergienko, and M. C. Teich, Phys. Rev. A 62, 043816 (2000).

19. A. F. Abouraddy, M. B. Nasr, B. E. A. Saleh, A. V. Sergienko, and M. C. Teich, Phys. Rev. A 65, 053817 (2002).

20. A. G. Van Engen, S. A. Diddams, and T. S. Clement, Appl. Opt. 37, 5679 (1998).

21. R. Tripathi, N. Nassif, J. S. Nelson, B. H. Park, and J. F. de Boer, Opt. Lett. 27, 406 (2002). 